\title{
The Application of Statistical Process Control in Quality Management
}

\author{
Weiping $\mathrm{Li}^{1, \mathrm{a}}$ \\ ${ }^{1}$ Luohe Medical College, Luohe, Henan, 462000 \\ a email
}

Keywords: Rural Elderly; Economic Burden of Disease; Influencing Factors

\begin{abstract}
In this paper, the use of survey data in 26 counties in Henan province 740 rural elderly, empirical analysis of the economic burden of disease and its influencing factors of rural elderly. The results show that the economic burden of the elderly in rural areas of disease depends on the severity of the disease, family income level and whether to participate in Medicare. This article is certainly an important contribution to the new rural cooperative medical insurance in reducing the economic burden of the elderly hospitalized, but at the same time We found that rural economic burden of disease and the elderly family economic situation presented upside down, low-income elderly bear higher health care costs and economic burden, therefore, public health policy and health care reform at this stage should pay more attention to health equity.
\end{abstract}

\section{Introduction}

The sixth Chinese census shows that China's population aged over 60 is about 178 million, accounting for 13.26 percent of China's total population, the population aged over 65 was 119 million, accounting for 8.87 percent of China's total population. Compared with the fifth Chinese census, the proportion of China 60 years of age and over 65 years man-days is increased by 2.93 and 1.91 percentage points. A large elderly population, especially senior citizens population increases, cause sub enormous economic and social pressures. World Health Organization (referred to as "WHO") (2000) study shows that the elderly • straight plays in social development in an important role, they continue to work voluntarily in the form of formal or informal, to help care for children and families, to teach young people production technology and life experience. Since the 1990s, many scholars from economics, sociology and public administration point of view of the aging population and bring a series of economic and social problems have been studied, they investigated the changes in their body functions and relationship between socio-economic factors, the elderly health and social support (such as Mr. Li, 2004), child care (for example, Chang Chen, 2004) and intergenerational support on older people's health and quality of life (such as Zhang Wenjuan, plum Zhuo, 2004 ), the aging trend of the age distribution of the rural medical costs (such as Yan Jun, Chen Yuping, 2010), as well as the impact of aging (for example, more than central central, 2011) of Chinese urban and rural medical expenses. Numerous studies show that the body is not only reflected in the quality of life of older persons health, but also to promote the necessary conditions for older people to continue to participate in social work. In China demographic dividend is disappearing in the background, by examining the economic burden of the elderly, thereby improving the health of the elderly to develop relevant policies, has a positive practical significance.

Unlike developed countries, China's aging population has an important feature of the urban and rural level inverted. The sixth Chinese census data show that China's rural population, elderly dependency ratio was 22.75 percent, 7.02 percentage points higher than the city. Compared with the city, the economic burden of the elderly in rural China issues more severe. According to the fourth national health services survey data, bi-rural elderly aged 60 and over prevalence of $37.8 \%$, 38.9\% prevalence of chronic diseases. The disease to the patient's family brought a severe economic burden on most families unbearable catastrophic medical expenses, $26.3 \%$ of the elderly should be hospitalized without hospitalization, give up the treatment. "How scientific analysis of rural elderly disease economic burden and ease that burden by what means, effectively improve the health status of the elderly in rural areas, is the main problem of this article. this article intends to use the County (cities, districts) of rural elderly sample survey data to study rural economic status of the elderly 
disease burden, rule and influential factors, and analyzed under the new rural cooperative medical insurance and comprehensively promote the background of the economic burden of the elderly in rural areas have been eased if, if their health has improved and so on.

\section{The Variable Selection and Model Form}

In this paper, the elderly live this close to a doctor treating the case as a miasma research object, first analyze what the elderly better able to withstand the economic burden of the disease, that the probability of treatment of the elderly and its influencing factors; Second degree analysis of the economic burden of disease and its influencing factors, the economic burden of the disease, including the direct costs arising from the treatment, and the total cost includes direct costs and indirect costs, including, at the same time use of medical expenses and household income per capita relative value to analyze the cost of treatment to the family degree of economic burden. In all the study protocol, the economic burden of disease burden and economic miasma were hospitalized economic burden from two perspectives to analyze the door. Due to the use of multiple indicators to represent the economic burden of disease variables, so a lot of the analysis section of this article involving multiple mode rejection and equations. Model and a second model for the elderly bear the economic burden of disease models, using binary Logit model analysis, the Che dependent variable is whether the elderly hospitalized for treatment and whether to use the service.

In this paper, factors that will affect the economic burden of the elderly disease are divided into the following four categories: individual and family characteristics, severity of disease, per capita household income, regional economic conditions. Among them, the severity of the disease and the economic situation is the main factor affecting the economic burden of disease in the elderly. Are the elderly sick and whether the disease is serious, directly determine whether the elderly can bear the economic burden of disease. In this paper, respondents to the subjective judgment of disease severity index ( "slight", "moderate" and "severe") to represent. Economic status variables are divided into two categories family income and local economic conditions. In this paper, according to respondents per capita household income quintile to low-income groups, low-income groups, middle-income groups, high-income group and high income groups, to reflect family income. Taking into account the northern Henan, Henan, the three regions south of Henan economic development and development of medical and health services there is a big difference in the economic situation variable region is set to dummy variable to reflect the regional economic situation and regional differences. In addition, gender, age, education, marital status, living conditions, chronic disease, whether to participate in individual and family health insurance and other basic characteristics as control variables into the model.

\section{The Model Estimation Results and Analysis}

Economic burden of disease regression results show a probability model, the severity of disease if treatment of the elderly negative impact on, and passed the test of significance; the impact of family income for the elderly whether treatment is also negative, where the low-income group and high-income groups through the test of significance 'indicates that these two groups of older people in a consultation on the elderly and other groups the differences are obvious. With all other variables held constant, the more severe the disease, the elderly in rural areas access to regular medical treatment to produce a higher probability of disease economic burden. In general, the severity of the disease is an important factor in determining whether a patient's treatment. The elderly feel more serious illness, the stronger the demand for medical services and more urgent. This result is corroborated to some extent there is a causal relationship between the health of older persons in this vulnerable population, disease and poverty.

The regression results hospitalized economic burden of probability models show, junior high school and higher education affect older persons are hospitalized for positive, and passed the test of significance. This is because the elderly have a higher educational level higher probability of suffering from a serious illness requiring hospitalization, elderly people with higher education have 
higher economic dominance, more emphasis on health, thus contributing to their inpatient care It uses. Whether the severity of the disease in elderly people hospitalized for negative and adopted a significant test. Elderly people suffering from more severe disease, the more we need to stay in hospital for treatment or observation, thereby increasing the probability of inpatient care use. High-income group variables that affect older persons are hospitalized for positive, and passed the test of significance. This is because the higher the probability of high-income groups and elderly people suffering from a serious illness requiring hospitalization, hospitalized after their sick conditional 'low-income groups and the elderly tend to be hospitalized due to financial difficulties or unwilling to give up hospitalization.

Data show that the economic burden of the elderly outpatient and inpatient model economic burden 8 regression model results. Gender, single elderly outpatient economic burden are negative. This shows that there is a clear gender differences in outpatient costs and outpatient economic burden, women's outpatient costs more outpatient heavier economic burden than men than men, because women in old age are biologically more vulnerable than men, resulting in women treatment costs and economic burden of disease is heavier than males. Therefore, the Government health policies should pay more attention to women's health and their health care. Marital status Single elderly, less its outpatient expenses. This is because the elderly if single, would be worried sick if lack of family support, which usually pay more attention to maintaining a healthy and active prevention. It is easy to understand, the more severe the disease, the more complex the required examinations and treatment, the more severe the economic burden of disease naturally. The more developed regions and the development level of Henan middle of rural elderly in northern regions of the cost of outpatient clinics and economic burden is higher than the economically underdeveloped areas of Susie. Whether to participate in the impact on the elderly Medicare outpatient pocket expenses and the total cost is negative, and passed the test of significance. This is due to the contribution of the new rural cooperative medical insurance is now widely implemented to reduce the elderly outpatient medical costs. Different income levels of the elderly, their outpatient medical expenses no significant difference, indicating that the elderly outpatient costs not linked with the family economic level, reflecting the medical expenses equitable distribution among different income groups of the elderly. However, further analysis of the ratio of outpatient medical costs and household income per capita burden of this economic indicator, found family income for the elderly outpatient copayment

With a negative impact of the financial burden and economic burden of the total cost, and. We have passed the test of significance, indicating the presence of the economic burden of outpatient significant differences among different income groups aged, the higher the household income, the more light-patient financial burden. This implies that the economic burden of disease unfair between different income levels of families, low-income elderly people bear a higher financial burden.

Whether to participate in medical insurance in elderly people hospitalized economic burden of negative and thus had medical insurance significantly reduces the cost of hospitalization of older persons, while other factors are not important determinants of hospitalization costs. Government's objective is to implement the new rural cooperative rural residents' health and reduce the economic burden of disease of rural residents, especially to reduce the large hospital medical expenses. The results of this estimation model demonstrates the NCMS important contribution in reducing hospitalization costs in rural areas the elderly, the effect of the implementation stage proved NCMS, also illustrates the NCMS important to reduce the economic burden of disease of older persons contribution. The impact of disease severity hospitalized economic burden is positive, and passed the test of significance. Family income affect the economic burden of hospitalized elderly are negative, the higher the per capita household income, the more light the elderly hospitalized economic burden. This reflects the economic burden of hospitalization among different income groups in vertical unfair, low-income elderly households suffered more serious economic burden. Since low-income family's economic vulnerability of the elderly, once the large medical expenses occur, and they are likely to poverty caused by illness. 
In summary, the severity of the disease is an important factor in rural economic burden of the elderly and economic burden of disease occurrence probability of the size, the more serious their illness, more outpatient pocket costs and total cost of outpatient and inpatient economic burden of the economic burden more severe. However, the economic burden of disease differences between income groups significantly older, and was "upside down" phenomenon. The medical people the principle of fairness, have the same level of income or ability to pay bear the same medical expenses, we can achieve the level of medical fair; high ability to pay people to pay higher medical costs, low capacity to pay people pay less medical expenses, will be able to reach medical vertical equity. Positive results of this analysis showed that the higher the level of per capita household income households, the elderly and hospital outpatient economic burden of the economic burden of more light: the lower the per capita household income level of the family, the more severe the economic burden the elderly outpatient and inpatient economic burden.

This shows that the economic burden of disease and the presence of areas surveyed elderly household per capita income related unfair. From the regional differences of view, the economic burden of the elderly probability of occurrence, hospital costs, hospital financial burden did not differ significantly between the south of Henan, Henan, the three regions in North, but the financial burden clinics in the rural areas of Henan elderly heavier. In addition, the results show that health insurance can effectively reduce the cost of outpatient, inpatient hospital costs and economic burden. Medicare is equivalent to the price of variables into the model, the elderly by health insurance, sharing economic risk of disease, significantly reducing the cost of outpatient and hospital costs, but also to ease the financial burden of hospitalization. This is an important contribution to the new rural cooperative medical care in China is currently widely implemented.

\section{Conclusions and Policy Implications}

Poverty eradication, to stay healthy and in good physical function, quality of life is the most important manifestation of the elderly, but also in the current demographic changes, the degree of aging deepening background, social cope with the negative impact of population aging will be focusing on the most basic issues. This paper shows that with the rural elderly age, his health declined, their medical expenses has become an important personal and family expenses, especially when seriously ill and urge the use of inpatient care, the disease will give the elderly and their families serious economic burden. Although the age can be seen as the depreciation of the body, but after controlling for other factors, the economic burden of the elderly with age there is no significant correlation, the most important factor is the severity of the disease, family income, whether to participate in medical insurance Wait.

The economic burden of disease among rural elderly household income groups were significant, indicating the presence of family income per capita health-related unfair. Whether the probability, or the size of the economic burden of disease, the lower the per capita household income of the elderly occur from the disease, the more severe the burden, which is contrary to the principles of health and health equity fair. Therefore, in the New Government should formulate public health policy and promote rural health care reform in focus to meet the demand for medical services in rural elderly people and promote health equity. Moreover, the empirical results of this study demonstrated that health insurance can reduce health care costs and economic burden of rural elderly generated in the course of treatment, particularly for the elderly to reduce hospitalization costs and reduce the economic burden of the elderly hospitalized plays an important role. This fact confirms the new rural cooperative medical care for nearly 10 years, the widespread implementation of the policy in effect to alleviate the economic burden of the rural elderly diseases and important contribution.

\section{Acknowledgements}

This is the Science and Technology Department of Henan Province soft science research project planning fiscal 2015. Project No.. 152400410031. 


\section{References}

[1] Beydoun, M. A. and Popkin, B. M .: The Impact of Socio-economic Factors on Functional Status Decline among Community-dwelling Older Adults in China, Social Science \& Medicine, Vol. 60, No9, pp. 2045-2057,2005.

[2] World Health Organization: Social Development and Aging: Crisis or Opportunity, http://www.who.int, 26 June, 2000.

[3] Gu Danan; "Suppliers age old personal social life Jie Nya economic characteristics 41 Dynamic Change of processing power" and the "H population science" 2004 Supplement.

[4] Li Jianxin: "Research on the relationship between social support and life satisfaction of the elderly U", "in the boudoir Population Division Bian" 2004 Supplement.

[5] Hui Suo, Chen Yuping: "ffl rural elderly accounted for more medical resources yet?", "Management World" 20105.

[6] Yu Yangyang: "the impact of aging on the WK treatment fee W - the urban-rural differences in perspective", "World Economic Forum" 20115.

[7] Zhang Chenchen, Li Shuzhuo: "on behalf of Shi support Impact on physical and mental health providers elderly age status flag", "China Population Science" 2004 Supplement.

[8] Zhang Dai: "Children living on the elderly health care: to promote or choose", "China Population Science" 2004 Supplement. 